Revue internationale P.M.E.

Économie et gestion de la petite et moyenne entreprise

\title{
L'impact des tâches critiques du gestionnaire de PME sur le succès d'un système d'information organisationnel
}

\section{Louis Raymond}

Volume 1, numéro 1, 1988

URI : https://id.erudit.org/iderudit/1007875ar

DOI : https://doi.org/10.7202/1007875ar

Aller au sommaire du numéro

Éditeur(s)

Presses de l’Université du Québec

ISSN

0776-5436 (imprimé)

1918-9699 (numérique)

Découvrir la revue

Citer cet article

Raymond, L. (1988). L'impact des tâches critiques du gestionnaire de PME sur le succès d'un système d'information organisationnel. Revue internationale P.M.E., 1(1), 77-95. https://doi.org/10.7202/1007875ar
Résumé de l'article

Cet article présente les résultats d'une étude empirique de la relation entre le travail managérial et le succès des systèmes d'information organisationnels (SIO) en contexte de PME. Utilisant la théorie des rôles managérials élaborée par Mintzberg, nous examinons l'impact du profil des tâches critiques du gestionnaire sur son utilisation d'un SIO, et sur l'aide qu'il en obtient. Compte tenu de certains attributs du gestionnaire et du système, le succès actuel des SIO dans la PME se concrétise par le soutien des tâches informationnelles des gestionnaires à l'aide de rapports imprimés, plutôt que par un soutien interactif des tâches décisionnelles. Nous discutons de ces résultats et de leurs retombées. 


\title{
L'impact des tâches critiques du gestionnaire de PME sur le succès d'un système d'information organisationnel*
}

\author{
Louis RAYMOND \\ Professeur agrégé, GREPME \\ Université du Québec à Trois-Rivières
}

\begin{abstract}
RESUME
Cet article présente les résultats d'une étude empirique de la relation entre le travail managérial et le succès des systèmes d'information organisationnels (SIO) en contexte de PME. Utilisant la théorie des rôles managérials élaborée par Mintzberg, nous examinons l'impact du profil des tâches critiques du gestionnaire sur son utilisation d'un SIO, et sur l'aide qu'il en obtient. Compte tenu de certains attributs du gestionnaire et du système, le succès actuel des SIO dans la PME se concrétise par le soutien des tâches informationnelles des gestionnaires à l'aide de rapports imprimés, plutôt que par un soutien interactif des tâches décisionnelles. Nous discutons de ces résultats et de leurs retombées.
\end{abstract}

\begin{abstract}
This article presents the results of an empirical study on the relationship between managerial work and the success of management information systems (MIS) in the context of small and medium-sized business (SMB). Using the theory of managerial roles developed by Mintzberg, we examine the impact of the manager's critical tasks profile upon his MIS usage, and upon the support he obtains from it. Taking into account some manager and system attributes, the present success of MIS in small business is defined by the support of the managers' informational tasks through printed reports, rather than by an interactive support of the decisional tasks. These results and their implications are discussed.
\end{abstract}

\section{RESUMEN}

Este articulo presenta los resultados de un estudio empirico de la relacion entre el trabajo empresarial y el éxito de los sistemas de informacion organizacionales (SIO) en contexto de pequenas y medianas empresas (PME). Utilizando la teoria de los roles empresariaes elaborada por Mintzberg, examinamos el impacto del perfil de las tareas criticas del gestor sobre su utilizacion de un SIO, y sobre la ayuda conseguida. Teniendo en cuenta ciertos atributos del gestor y del sistema, el éxito actual de los SIO en la PME se concreta por el apoyo de las tareas informacionales de los gestores con rendimientos imprimidos, antés que por un apoyo interactivo de los tareas decisionales. Discutimos estos resultados et sus recaidas.

* La recherche a été subventionnée par l'Institut de recherches politiques (Québec). L'auteur tient à remercier le rédacteur en chef et les évaluateurs anonymes dont les critiques et les suggestions ont grandement contribué à l'amélioration de cet article. 


\section{Introduction}

Plusieurs facteurs ont été reliés au succès des systèmes d'information dans les organisations. Une énumération représentative mais non exhaustive de ceux qui ont été étudiés empiriquement inclut les facteurs de contexte organisationnel, les caractéristiques individuelles des utilisateurs et les caractéristiques techniques des systèmes et de l'interface homme-machine. La plus forte part de ces recherches est centrée sur l'influence déterminante des «différences individuelles», reliées à la tâche, au style cognitif, à la personnalité et aux attitudes (Baile, 1985; Zmud, 1979), sur l'implantation et l'utilisation d'un système d'information organisationnel à base d'ordinateur(SIO). On doit noter cependant que les études empiriques del'interaction SIO-utilisateur ont surtout été effectuées dans un contexte de grande entreprise. Or, plusieurs chercheurs ont confirmé la spécificité des gestionnaires de PME sur divers plans tels que la tâche (Paolillo, 1984), la personnalité (Harrell, 1971) et les comportements de gestion et de prise de décision (Fallery, 1983; Gasse, 1979; Young et Welsch, 1983). Ainsi, les résultats de recherches sur les gestionnaires de grandes entreprises en tant qu'utilisateurs de systèmes d'information ne peuvent être directement extrapolés à la PME. D'où la nécessité d'études spécifiques à ce contexte. Cela est d'autant plus important que les SIO peuvent actuellement être considérés comme l'un des facteurs critiques du succès de la PME (Dickinson, Ferguson et Sircar, 1984).

Etant donné que l'objectif primordial d'un système d'information organisationnel est d'assister le gestionnaire dans ses fonctions de planification, de contrôle et de prise de décision (Davis et Olson, 1985), la nature et l'environnement de la tâche de ce gestionnaire sont des facteurs cruciaux qui doivent entrer en ligne de compte lors de la conception et de l'implantation d'un tel système. Sans quoi le SIO ne sera pas utilisé par le gestionnaire, ou ne lui apportera pas de soutien efficace (Poole, 1978). Dans cet ordre d'idées, notre recherche a pour but d'explorer l'impact sur le succès d'un SIO, de différences individuelles au niveau de la tâche des gestionnaires de PME'

Pour ce faire, nous utiliserons une conceptualisation reconnue de la nature du travail managérial, soit celle d'Henry Mintzberg (1973a). Quoique les travaux de Mintzberg soient souvent cités comme fondement théorique de la recherche en systèmes d'information, son concept du rôle managérial n'y a jamais été utilisé de façon opérationnelle.Les chercheurs ont plutôt tenté de cemer l'impact des différences de rôle et de tâche des utilisateurs à travers des variables telles que le niveau d'ambiguîté et de conflit du rôle, la complexité et l'identité de la tâche, la variabilité de l'environnement de prise de décision et le niveau d'autonomie (Culnan, 1983; Sanders et Courtney, 1985; Saunders et Keller, 1983).

1 «Gestionnaire» inclut ici non seulement le dirigeant mais tout employé qui occupe un poste de gestion dans la PME. 
A chaque fonction ou position dans l'organisation est associé un ensemble d'activités ou de comportements attendus. Ces activités constituent le rôle qui doit être tenu, du moins approximativement, par tout individu qui occupe cette fonction (Katz et Kahn, 1978). Selon Mintzberg (1973a), la gestion d'une organisation dans un environnement complexe implique qu'un individu joue une série de rôles managériaux, et les exigences de ces rôles induisent des caractéristiques qui sont communes à la tâche de tout gestionnaire.

Katz et Kahn (1978) notent que des attributs individuels tels que l'expérience et la personnalité ne déterminent pas si un rôle est joué ou non, mais ils peuvent influencer la manière dont le rôle sera joué. Notre étude tentera donc d'évaluer la pertinence du concept de rôle managérial pour la recherche en systèmes d'information dans le contexte de la PME. Plus précisément, il s'agit de mesurer et d'analyser l'impact des diffẹ́rences individuelles relatives aux divers rôles joués par les gestionnaires de PME, d'une part sur les comportements de ces derniers face à un SIO, et d'autre part sur le niveau de soutien qui leur est fourni par le système.

\section{Cadre conceptuel}

Le modèle de recherche utilisé dans notre étude est présenté à la figure 1. Les variables indépendantes sont celles qui décrivent le profil de la tâche du gestionnaire en termes de rôles managérials. Ces variables ont un impact présumé sur le succès d'un SIO en termes de l'utilisation qu'en fait le gestionnaire, et du soutien qu'il en retire (variables dépendantes). Cependant, certains résultats d'études antérieures nous portent à croire que la nature de la tâche du gestionnaire de PME ne peut à elle seule expliquer totalement ses comportements envers un SIO; en particulier, l'environnement individuel de la tâche, en termes d'attributs acquis par le gestionnaire tels que l'expérience et la formation, doit aussi être considéré (Taylor et Dunnette, 1974). De plus, certains attributs du SIO, constituant l'environnement organisationnel immédiat de l'utilisateur, et en particulier ceux qui caractérisent le portefeuille d'applications de la PME, sont aussi de nature à conditionner l'utilisation et le soutien d'un système informatisé (Raymond, 1987). 
Fig. 1 Modèle de recherche reliant le profil des tâches critiques du gestionnaire de PME au succès du SIO

\begin{tabular}{|l|c|}
\hline \multicolumn{1}{|c|}{$\begin{array}{c}\text { ATTRIBUTS DE } \\
\text { L'UTILISATEUR }\end{array}$} \\
\hline $\begin{array}{l}\text { - Expérience informatique } \\
\text { - Formation informatique }\end{array}$ \\
\begin{tabular}{|l|l|}
\hline $\begin{array}{l}\text { PROFIL DES TACHES CRITIQUES } \\
\text { DE L'UTILISATEUR-GESTIONNAIRE }\end{array}$ & SUCCES DU SIO \\
\hline - Nombre de tâches informationnelles & - Utilisation (rapports) \\
- Nombre de tâches décisionnelles & Utilisation (dialogué) \\
- Nombre de tâches critiques \\
soutenues par le SIO
\end{tabular} \\
\hline
\end{tabular}

\subsection{Profil des táches critiques du gestionnaire de PME}

A partir de ses observations de gestionnaires au travail, Mintzberg (1973a) a identifié un ensemble de dix rôles managérials, regroupés et définis succinctement de la façon suivante :

Rôles informationnels

- contrôleur («monitor», recherche et obtention d'informations par des média de communication verbale ou non verbale)

- diffuseur («disseminator», transmission d'informations aux subalternes)

- porte-parole («spokesperson», transmission d'informations à l'extérieur du groupe de travail). 


\section{Rôles décisionnels}

- répartiteur de ressources («resource allocator», répartition des ressources entre les différentes unités organisationnelles)

- entrepreneur (recherche d'opportunité et planification de nouvelles activités pour améliorer la performance)

- redresseur («disturbance handler», actions correctives pour ce qui touche aux problèmes et aux pressions)

- négociateur («négociator», négociation avec les employés, les clients, les fournisseurs et les syndicats)

\section{Rôles interindividuels}

- leader (motivation, orientation et développement des subalternes)

- figure de proue («figurehead», activités nécessitant des devoirs cérémoniels, sociaux ou juridiques)

- agent de liaison («liaison», maintien de contacts avec des individus non subalternes)

On peut ainsi associer un rôle managérial particulier à chaque activité ou tâche du gestionnaire.

Des tests empiriques du modèle de Mintzberg ont confirmé sa validité (Kurke et Aldrich, 1983; Shapira et Dunbar, 1980), c'est-à-dire que ces dix rôles semblent effectivement communs au travail de tous les gestionnaires. On notera cependant que Paolillo (1984) a trouvé des différences à ce niveau en fonction de la taille des entreprises, les rôles d'entrepreneur et de leader étant relativement plus importants pour les gestionnaires de PME. De plus, Alexander (1979) a confirmé la présence d'une certaine spécialisation des rôles selon la fonction occupée par le gestionnaire, les responsables de la production, des ventes et du personnel de soutien tendant à privilégier respectivement les rôles décisionnels, interindividuels et informationnels.

Or, la question centrale qui nous préoccupe est de savoir si l'existence de différences quant à l'importance relative accordée par les gestionnaires de PME à leurs divers rôles managérials, peut affecter le succès d'un SIO. Une meilleure compréhension de l'interaction entre la tâche de l'utilisateur et son comportement envers un système d'information devrait nous conduire à une conception de systèmes plus efficaces, parce que mieux adaptés à la nature de cette tâche. C'est ainsi que l'on peut présumer qu'un gestionnaire dont la tâche privilégie les rôles interindividuels aura un faible niveau d'utilisation du système très formalisé qu'est un SIO, préférant s'appuyer surun systèmed'information informelet personnel plutôtqu'organisationnel (Mintzberg, 1973b). A partir d'observations du travail de dirigeants de PME, Fallery (1983) a trouvé que le comportement managérial propre à ces derniers est caractérisé 
par la variété, la brièveté, la faiblesse du temps de «travail personnel» et par une part très forte de tâches de communication; il en conclut que la logique de ce comportement est peu compatible avec la logique actuelle de fonctionnement des SIO. A l'inverse, la nature des rôles informationnels les associe directement à la fonction du SIO qui est de fournir au gestionnaire de l'information opportune, pertinente, précise et complète. Par exemple, les individus dont la fonction principale est d'obtenir et de diffuser l'information pour la planification et le contrôle budgétaires et financiers devraient être de plus gros «consommateurs» d'un SIO (Trahand, 1983). On peut donc penser que les gestionnaires qui privilégient les rôles informationnels obtiendront un soutien plus important de la part d'un système qui produit de l'information plus complexe et plus structurée.

L'impact d'une tâche managériale dominée par les rôles décisionnels est cependant plus difficile à prévoir, étant donné certaines caractéristiques spécifiques au gestionnaire de PME et à son environnement. D'une part, des recherches antérieures ont démontré que le modèle néo-rationnel de la prise de décision (Simon, 1960), axé sur l'acquisition et le traitement de l'information, est inapproprié dans ce contexte (Paulson et Stup, 1979). Dans une étude empirique sur la prise de décision dans la PME (Rice et Hamilton, 1979), les gestionnaires indiquèrent en effet que la majorité de leurs décisions résultaient de «l'expérience», de «l'intuition», ou étaient effectuées «au jugen». Tibbits (1979) ainsi que Chicha et Julien (1980) ont confirmé ce point de vue en affirmant que les décisions sont souvent prises après une analyse moins détaillée que dans les grandes entreprises. Il peut donc en résulter une sousutilisation des SIO dans ce contexte (Massey, 1986). D'autre part, l'émergence du micro-ordinateur et des logiciels de 4ième génération tels que les tableursélectroniques (Hoppen et Trahand, 1985) permettrait éventuellement au gestionnaire de PME de disposer de systèmes interactifs d'aide à la décision relativement faciles à concevoir et à utiliser (Cooley, Waltz et Waltz, 1987). L'évolution des systèmes d'information tend en effet à accentuer les capacités de repérage et d'analyse à partir d'un dialogue homme-machine, et ce, aux dépens des systèmes traditionnels axés sur le contrôle à partir de rapports périodiques ou d'exception(Alloway et Quillard, 1982). Dans une optique normative, cette évolution devrait amener les gestionnaires de PME qui utilisent actuellement un SIO à être plus efficaces dans l'accomplissement de leurs rôles décisionnels.

Lors de l'enquête sur le terrain effectuée pour fins de cette recherche, nous avons demandé à des individus de décrire les tâches qu'ils jugeaient les plus critiques à leur succès en tant que gestionnaire et au succès de leur entreprise. A partir de cette description, l'intervieweur devait alors assigner un rôle managérial à chaque tâche en fonction de la typologie de Mintzberg (1973a). La distribution résultante, en termes du nombre de tâches critiques assignées respectivement à des rôles informationnels, décisionnelset interindividuels, nous fournit ainsiune caractérisation appropriée de la tâche d'un gestionnaire de PME en fonction de l'objectif de recherche. 


\subsection{Attributs de l'utilisateur-gestionnaire}

Un certain nombre de caractéristiques socio-démographiques telles que l'âge, le niveau hiérarchique, la scolarité et l'expérience pourraient possiblement affecter les attitudes et les comportements des utilisateurs d'un systèmed'information, bien que les résultats empiriques sur ce plan aient été plutôt mitigés (Zinkhan, Joachimsthaler et Kinnear, 1987). Etant donné l'objectif de recherche, deux de ces caractéristiques furent incluses dans le modèle de recherche en tant que variables modératrices et ce, dans le but d'accroître la fiabilité des résultats. Ces attributs, soit l'expérience et la formation informatiques du gestionnaire, furent choisies en raison de la non-ambiguité et de l'importance de leur relation théorique et empirique avec le succès des SIO, telles qu'établies par des études antérieures réalisées en contexte de PME.

Un gestionnaire de PME qui a une plus grande expérience des systèmes informatisés est plus familier avec leur mode d'opération, et avec le type de soutien que ces systèmes peuvent lui apporter dans l'accomplissement de ses tâches. De cette familiarité devrait résulter une plus grande utilisation d'un SIO (Nickel et Seado, 1986). Un argument similaire peut être invoqué relativement à la formation informatique, cette dernière agissant alors comme substitut ou complément de l'expérience, particulièrement dans le cas du gestionnaire de PME dont l'expérience informatique est souvent limitée (Raymond, 1988). Cette dernière est mesurée par le nombre d'années depuis lequel l'utilisateur a recours à des systèmes informatisés pour fins de gestion. La formation informatique est mesurée de façon dichotomique ( 1 : présence, $0:$ absence) en déterminant si le gestionnaire a suivi au moins un cours formel provenant de sa formation universitaire ou collégiale, d'un fournisseur ou d'un organisme spécialisé.

\subsection{Attributs du SIO}

Il apparaît de façon assez évidente que la nature des applications du SIO qui ont effectivement été implantées par une PME constitue une contrainte fondamentale sur le comportement de l'utilisateur dans sa recherche de l'information et sur le niveau de support informationnel obtenu. Cela est d'autant plus vrai que le gestionnaire de PME occupe généralement une fonction dont l'envergure est étendue, à la fois verticalement (contrôle opérationnel, contrôle de gestion, planification stratégique) et horizontalement (marketing, production, finance, ressources humaines). Or, des études empiriques ont démontré que l'exploitation d'un plus grand nombre d'applications «de gestion»², c'est-à-dire d'applications de soutien des fonctions de planification, de contrôle et de prise de décision, est associée à des plus hauts niveaux

2 Par opposition à des applications «administratives» de soutien des opérations de l'entreprise (traitement des transactions). 
de satisfaction et d'utilisation du SIO (Raymond, 1985) et à un meilleur soutien des tâches critiques du gestionnaire (Alloway et Quillard, 1982). De même, plusieurs recherches ont confirmé que la facilité d'accès, la réduction du volume des sorties et le court temps de réponse associés au mode d'exploitation «en dialogué» ${ }^{3}$, soit la possibilité d'interagir directement avec une application à partir d'une console, ont un impact positif sur les gestionnaires en tant qu'utilisateurs de SIO (Dickson, Senn et Chervany, 1977; Raymond, 1985). Le nombre d'applications dont l'objectif prépondérant est le soutien de la gestion, et le nombre dont le mode d'exploitation prépondérant est en dialogué, seront donc utilisés comme mesures caractéristiques du SIO d'une entreprise.

\subsection{Succès du SIO}

Trois mesures substitutives du succès d'un SIO sont employées dans la recherche. Les deux premières reflètent le comportement d'utilisation du gestionnaire en termes de la fréquence de consultation de rapports imprimés et du nombre d'accès à partir d'une console (Srinivasan, 1985). La troisième mesure est indicative du niveau de soutien fourni parle SIOàl'utilisateur, elle fut obtenue suiteàl'identification des tâches critiques du gestionnaire, en lui demandant d'indiquer celles qui étaient assistées de quelconque façon par le SIO (Alloway et Quillard, 1982). Notons que ces variables sont considérées comme substitutives dans la mesure où les véritables indicateurs de succès d'un SIO seraient la performance du gestionnaire, et ultimement la performance de l'entreprise. Or, compte-tenu de la difficulté d'obtenir des mesures adéquates de l'efficacité individuelle et organisationnelle dans un contexte de recherche sur le terrain (Trice et Treacy, 1986), on considérera ici qu'un SIO a d'autant plus de succès qu'il est plus utilisé par les gestionnaires et qu'il les soutient dans un plus grand nombre de tâches critiques.

\section{$3 \quad$ Méthode de recherche}

Les données recueillies pour les fins de la recherche proviennent de $34 \mathrm{PME}$ manufacturières québécoises, soit 9 firmes d'un secteur plus traditionnel, celui du bois, et 25 firmes d'un secteur plus novateur, celui des produits métalliques. Le critère typologique le plus souvent employé pour distinguer une PME d'une grande entreprise dans le secteur manufacturier est le nombre d'employés de production; sur cette base, l'échantillon comporte 18 PE (6-50 employés) et 16 ME (51-250 employés). Toutes ont implanté un système d'information organisationnel à base

3 Par opposition au mode d'exploitation «par lots« (émission périodique de rapports imprimés) 
d'ordinateur, et ce, depuis trois ans en moyenne. Le secteur manufacturier fut choisi parce qu'on y retrouve actuellement un plus large éventail d'applications de l'informatique aux différentes fonctions de la PME. La cueillette des données fut réalisée au moyen d'entrevues semi-structurées à l'aide d'un questionnaire. Un total de 91 gestionnaires furent interviewés, incluant 29 dirigeants (PDG), 34 responsables de la fonction comptabilité-finance et 28 responsables de production. Les 34 responsables du SIO dans chaque firme furent aussi rencontrés.

Etant donné le modèle de recherche, des coefficients de corrélation ( $\mathrm{r}$ de Pearson) furent calculés pour déterminer les relations existant entre les variables. En particulier, le calcul de corrélations partielles permet d'éliminer statistiquement l'effet des variables modératrices sur le lien entre la tâche du gestionnaire et le succès du SIO (Nie et al., 1975). On notera que toutes les variables sont à échelle d'intervalles, à l'exception de la formation informatique qui est dichotomique; Nie et al. (1975) indiquent qu'à toutes fins pratiques, une dichotomie peut être utilisée en tant que mesure ordinale ou à intervalle. Quant à la distribution des variables, l'utilisation de statistiques paramétriques s'appuie sur la constatation de Kerlinger (1973) selon laquelle «à ce jour, les résultats confirment que l'importance de la normalité et de l'homogénéité est surestimée».

\section{Résultat et discussion}

Les statistiques descriptives des variables de recherche sont présentées au tableau 1. Dans un premier temps, ces résultats confirment l'existence de différences significatives en termes de l'importance relative accordée par les gestionnaires de PE à leurs divers rôles managérials, et ce, selon la fonction qu'ils occupent. C'est ainsi que les responsables de production tendent à privilégier les tâches de nature décisionnelle, alors que les tâches critiques des responsables financiers sont en très grande majorité de nature informationnelle (surtout de type «contrôleur»). Ces derniers sont effectivement surtout à la recherche d'informations pour détecter les changements à apporter, les problèmes ou les possibilités, et pour savoir quand des décisions doivent être prises. La tâche des PDG présente quant à elle la plus grande variété en matière de rôles de gestion, confirmant les propriétaires-dirigeants de PME dans leur vocation «d'homme-orchestre» de l'organisation. On notera la tendance générale des gestionnaires de PME à considérer les tâches interindividuelles comme étant moins critiques au succès de leur entreprise. 
Tab.1. Statistiques descriptives des variables de recherche

\begin{tabular}{|c|c|c|c|c|c|c|}
\hline $\begin{array}{l}\text { Variable } \\
\text { (n) }\end{array}$ & $\begin{array}{l}\text { Moy. } \\
\text { PDG } \\
(29)\end{array}$ & $\begin{array}{l}\text { Moy. } \\
\text { Fin. } \\
\text { (34) }\end{array}$ & $\begin{array}{l}\text { Moy. } \\
\text { Prod. } \\
\text { (28) }\end{array}$ & \multicolumn{3}{|c|}{$\begin{array}{l}\text { Gestionnaires } \\
\text { (91) }\end{array}$} \\
\hline Nbre de tâches informationnelles & 2,2 & 4,6 & 1,0 & 2,8 & 2,3 & 10 \\
\hline Nbre de tâches décisionnelles & 2,4 & 1,0 & 2,7 & 1,7 & 1,7 & 7 \\
\hline Nbre de tâches interindividuelles & 0,8 & 0,3 & 0,7 & 0,6 & 0,8 & 3 \\
\hline Expérience informatique (années) & 5,1 & 4,5 & 1,6 & 3,8 & 4,1 & 18 \\
\hline Formation informatique $(0,1)$ &, 31 &, 62 & ,25 &, 41 & - & 1 \\
\hline Niveau d'utililisation-rapports (1) & 9 & 29 & 13 & 18 & 23 & 96 \\
\hline Niveau d'utilisation-dialogué (2) & 1 & 13 & 3 & 6 & 13 & 48 \\
\hline Nbre de tâches critiques soutenues & 1,7 & 2,6 & 0,9 & 1,8 & 1,8 & 7 \\
\hline & $\begin{array}{l}\mathrm{PE} \\
(18\end{array}$ & & & $\begin{array}{l}\text { PME } \\
\text { (34) }\end{array}$ & & \\
\hline Nbre d'applications de gestion & 3 & &, 1 & 4,1 & 2,8 & 10 \\
\hline Nbre d'applications en dialogué & 3, & &, 9 & 3,9 & 4,2 & 15 \\
\hline
\end{tabular}

(1) Nbre moyen de consultations mensuelles

(2) Nbre moyen d'accès mensuels à partir d'une console

On retrouve aussi des différences significatives au niveau des attributs des gestionnaires en tant qu'utilisateurs d'un SIO. En effet, les responsables de production ont moins d'expérience informatique que les autres, alors que la proportion des gestionnaires qui ont reçu une quelconque formation informatique est plus forte dans le cas des responsables financiers. Ces derniers se distinguent en plus par une plus forte utilisation du SIO, particulièrement en ce qui a trait aux accès en dialogué. Il est intéressant de noter que lesPDG prétendent néanmoins obtenir un soutien presqu'aussi important que celui des gestionnaires financiers, ce qui pourrait être relié à une tâche plus variée ou à une utilisation plus sélective du SIO.

Conformément à des résultats antérieurs sur les liens entre la taille de l'organisation et les caractéristiques du SIO (Lehman, 1985; Raymond, 1985), lesPE ont implanté moins d'applications de gestion et moins d'applications en dialogué que les ME. Les autres différences significatives à ce niveau se limitent au fait que les 50 gestionnaires de PE ont plus de tâches critiques de nature décisionnelle (2,32 versus $1,56)$ et moins d'expérience informatique $(2,9$ années versus 4,8$)$ que les 41 gestionnaires de ME (test $\mathrm{t}$ bicaudal, $\mathrm{p}<0,05$ ). Toutefois, il fut vérifié que la taille n'avait aucun impact significatif sur la relation entre les tâches critiques du gestionnaire et le succès du SIO. 


\subsection{Impact des attributs de l'utilisateur et du SIO}

Les corrélations des variables modératrices avec les variables dépendantes du modèle de recherche sont présentées au tableau 2. L'impact global significatif des attributs de l'utilisateur et du portefeuille d'applications sur le succès du SIO, allié aux différences individuelles décrites précédemment, confirme la nécessité de tenir compte de ces variables dans notre analyse. Notons que ce sont les caractéristiques individuelles, et surtout la formation informatique, qui semblent avoir un effet primordial. Or, la formation des utilisateurs est un aspect souvent négligé lors de l'implantation d'un système d'information dans une PME. On comprend alors pourquoi, en PME, le volet «service» (formation, documentation, soutien technique) est tout aussi important au succès d'un SIO, sinon plus, que le volet «produit» (matériel et logiciel).

Tab. 2. Intercorrélations des variables dépendantes

et corrélations des variables modératrices avec les variables dépendantes

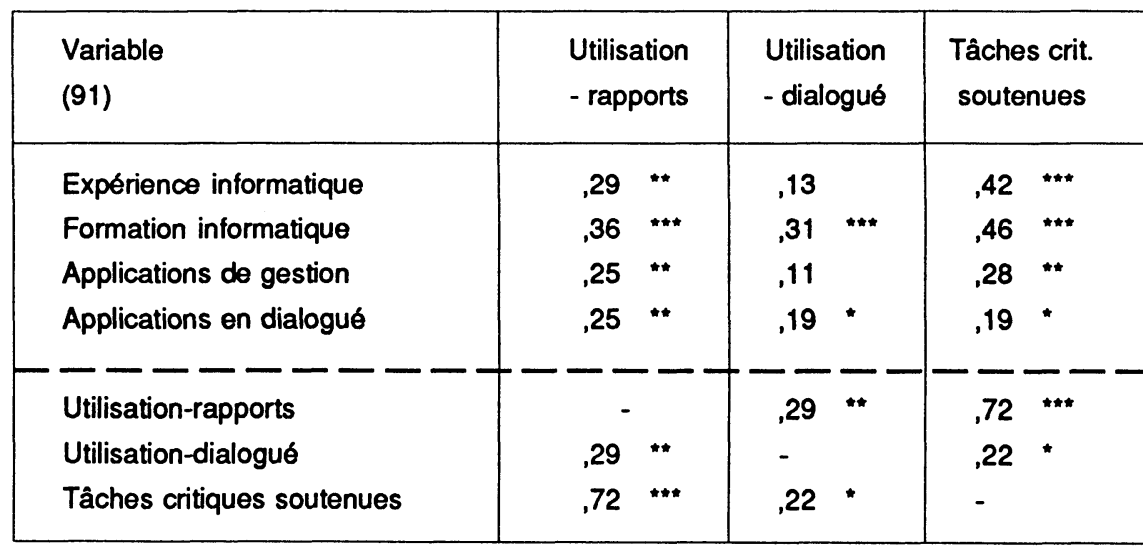

$$
\begin{aligned}
& \text { * } p<0,05 \\
& \text { ** } p<0,01 \\
& \text { ** } p<0,001
\end{aligned}
$$

On doit aussi constater que l'impact de ces attributs se fait surtout sentir sur les tâches critiques soutenues par le SIO et sur l'utilisation de rapports imprimés, l'utilisation en accès directn'étant reliée nià l'expérience informatique, ni au nombre d'applications de gestion. Cela pourrait indiquer qu'en regard de l'interaction directe avec le SIO, le processus d'apprentissage du gestionnaire de PME est conditionné 
plus par le contexte organisationnel et individuel d'utilisation (p.e. le manque de personnel de soutien), tandis que l'utilisation de rapports imprimés tend naturellement à croître au fur et à mesure que l'entreprise implante de nouvelles applications. D'ailleurs, bien que les trois variables dépendantes soient corrélées entre elles tel que prévu, le nombre de tâches critiques soutenues par le SIO est beaucoup plus relié à la consultation des imprimés du système qu'aux accès en dialogué. Ces résultats concordent donc avec la tendance encore prédominante des gestionnaires de PME à ne compter que sur le support passif d'un SIO, axé sur l'émission de rapports, plutôt que sur le support interactif à base de dialogues homme-machine.

\subsection{Impact des tâches critiques du gestionnaire}

La relation entre le profil des tâches critiques du gestionnaire et le succès du système d'information organisationnel peut être analysée à partir des coefficients de corrélation présentés au tableau 3. Une comparaison entre les parties gauche et droite du tableau révèle clairement l'effet modérateur des attributs du gestionnaire et du SIO sur cette relation. $L$ 'analyse qui suit sera donc basée sur les coefficients de corrélation de droite, c'est-à-dire ceux dont on a retiré statistiquement l'effet des variables modératrices pour plus de précision.

Les résultats démontrent clairement que les gestionnaires de PME dont les tâches critiques sont à prédominance informationnelle tendent actuellement à être de plus gros utilisateurs et à recevoir plus de soutien d'un SIO. C'est ainsi que l'on pourrait expliquer le fait que lesPDG et les responsables de production ont un niveau d'utilisation beaucoup plus faible que les responsables de la fonction comptabilitéfinance, et ce, indépendamment du fait que ces demiers ont plus de formation informatique. En effet, la majorité des tâches critiques des gestionnaires financiers sont associées au rôle informationnel de type contrôleur, ce terme étant d'ailleurs souvent employé comme tel pour identifier le poste occupé par ces individus. Leurs besoins, axés sur les opérations financières et le contrôle, seront plus facilement satisfaits par les applications présentement implantées dans les PME que des besoins axés sur les ventes, la production et la planification (Raymond, 1987). Notons aussi que le nombre de tâches informationnelles est plus fortement relié à l'utilisation de rapportsqu'àl'accès en dialogué, ce qui confirme la tendance actuelle des gestionnaires de PME quant à la façon dont ils exploitent leur SIO. 
Tab. 3. Corrélations des tâches critiques du gestionnaire de PME avec le succès du SIO

\begin{tabular}{|c|c|c|c|c|c|c|}
\hline \multirow[b]{2}{*}{$\begin{array}{l}\text { Variable } \\
\text { (91) }\end{array}$} & \multicolumn{3}{|c|}{$\begin{array}{l}\text { Sans contrôle des } \\
\text { var. modératrices }\end{array}$} & \multicolumn{3}{|c|}{$\begin{array}{l}\text { Avec contrôle des } \\
\text { var. modératrices }\end{array}$} \\
\hline & $\begin{array}{l}\text { Util.- } \\
\text { rapports }\end{array}$ & $\begin{array}{l}\text { Util.- } \\
\text { dialogué }\end{array}$ & $\begin{array}{l}\text { Tâches } \\
\text { soutenues }\end{array}$ & $\begin{array}{l}\text { Util.- } \\
\text { rapports }\end{array}$ & $\begin{array}{l}\text { Util.- } \\
\text { dialogué }\end{array}$ & $\begin{array}{l}\text { Tâches } \\
\text { soutenues }\end{array}$ \\
\hline $\begin{array}{l}\text { Nbre de tâches } \\
\text { informationnelles }\end{array}$ & , ** &, $22{ }^{*}$ &, $52 \cdots$ & , 28 &, $16^{\circ}$ & , $42^{* * *}$ \\
\hline $\begin{array}{l}\text { Nbre de tâches } \\
\text { décisionnelles }\end{array}$ & $-0,7$ &,$- 23 \cdot$ & $-19 *$ & $.01 \quad$ &,- 17 &,- 13 \\
\hline $\begin{array}{l}\text { Nbre de tâches } \\
\text { interindividuelles }\end{array}$ & -08 &,- 13 &,$- 21 \quad *$ & .02 &,- 09 &,- 10 \\
\hline - $p<0,05$ & & & & & & \\
\hline$\Rightarrow \quad p<0,01$ & & & & & & \\
\hline$* * \quad p<0,001$ & & & & & & \\
\hline
\end{tabular}

Etant donné la faible importance de la composante interindividuelle dans le profil des tâches critiques des gestionnaires de PME et la nature même des SIO dans ces entreprises, l'absence de résultats significatifs à ce niveau n'est pas surprenant. Cependant, il n'est pas dit qu'un SIO ne puisse pas offrir éventuellement une assistance au gestionnaire dans certaines tâches interindividuelles. Par exemple, l'utilisation appropriée d'applications de la bureautique (Moore, 1987) telles que le traitement de texte, le graphisme par ordinateur et le courrier électronique, pourraient contribuer à améliorer la performance de l'individu dont l'un des rôles managérials primordiaux est celui d'agent de liaison.

Finalement, les résultats démontrent que les gestionnaires de PME dont la tâche privilégie les rôles décisionnels tendent actuellement à être moins assistés par un SIO, et surtout à moins recourir au système de façon interactive. D'une part, cela confirme la théorie descriptive de la prise de décision des gestionnaires de PME, selon laquelle ces derniers effectuent peu de recherche et d'analyse d'informations avant de prendre une décision ${ }^{4}$. D'autre part, ces résultats sont contraires à une vision

4 Un phenomène similaire peut exister dans certains types de grandes organisations, où l'analyse de l'information ne servirait qu'à justifier une décision déjà prise (rationalité a posteriori, Crozier et Friedberg, 1977). 
normative d'un SIO dans l'entreprise, selon laquelle l'utilisation du système et surtout d'applications interactives d'aide à la décision, est censée rendre les gestionnaires plus efficaces dans l'accomplissement de leurs rôles décisionnels. Or, les PDG et les responsables de production, pour qui ces rôles prévalent, sont respectivement ceux qui utilisent le moins le SIO, et ceux qui sont le moins soutenus par le système. La sous-utilisation dans ce contexte pourrait s'expliquer par des raisons telles que la résistance au changement et l'aversion du risque, associées au manque d'adéquation entre la logique des systèmes actuellement implantés dans la PME et les processus de travail ou le style de prise de décision des gestionnaires (Dupuy, 1987). De plus, les avantages associés aux applications d'aide à la décision sont beaucoup moins tangibles au départ, et moins quantifiables que dans les cas des applications purement administratives, alors que ce sont les premières qui touchent véritablement le «coeur» de l'entreprise (Barcet, Bonamy et Mayère, 1984).

Enfin, les gestionnaires ne semblent pas encore considérer que l'informatique de l'utilisateur final («end-user computing») (Henderson et Treacy, 1986) pourrait combler certaines des lacunes actuelles des SIO en matière de soutien des tâches décisionnelles. Bien qu'originant des grandes entreprises, cette approche aurait le potentiel de s'adapter aux besoins de la PME en matière d'aide à la décision, et à ses contraintes en matière de ressources humaines et financières, étant donné les avantages suivants par rapport à l'informatique organisationnelle :

- un plus faible niveau d'investissement en matériel et en logiciel;

- une moins grande dépendance vis-à-vis des fournisseurs et des sociétés de services;

- une plus grande possibilité de développer des applications sur mesure (versus l'achat de progiciels);

- une plus grande possibilité de développer des applications de gestion et d'aide à la décision (versus administratives);

- un cycle de développement des applications plus court donc plus compatible avec l'horizon temporel de la PME;

- un moins grand recours au personnel spécialisé (informaticiens).

Or, seulement 8 des 91 gestionnaires échantillonnés ont eu recours à des outils micro-informatiquestels que des tableurs pour se générer un système «personnel» d'information ou d'aide à la décision (Snitkin et King, 1986). On doit donc conclure pour le moment que le succès des systèmes d'information dans la PME se concrétise par le soutien des tâches informationnelles des gestionnaires à l'aide de rapports imprimés, plutôt que par un soutien interactif des tâches décisionnelles. 


\section{$5 \quad$ Retombées et limites de la recherche}

Les résultats qui précèdent ont des retombées sur la recherche et la pratique des systèmes d'information. Sur le plan de la recherche, cette étude apporte une contribution additionnelle à une théorie descriptive des facteurs de succès des SIO dans la petite et moyenne entreprise. En particulier, les résultats tendent à confirmer la pertinence du concept des rôles managérials de Mintzberg en tant que fondement théorique pour appréhender la nature fondamentale de l'interaction utilisateursystème. Une emphase sur le travail managérial lui-même plutôt que sur des variables plus générales telles l'ambiguiité du rôle ou la complexité de la tâche pourrait donc nous permettre de mieux comprendre l'impact actuel et potentiel des systèmes d'information sur le gestionnaire de PME. C'est ainsi que l'on pourrait aborder la conception d'un système type en fonction de chaque rôle managérial, et, inversement, l'on pourraitétudier comment l'utilisation d'un SIO amène le gestionnaire à modifier l'importance relative accordée à chacun de ses rôles.

Sur le plan de la pratique, il pourrait être utile d'examiner le profil des rôles des gestionnaires durant la phase d'identification des besoins informationnels d'un projet de développement de système d'information. Il faudrait alors tenir compte de divers facteurs tels que la taille de l'entreprise et la fonction occupée, qui peuvent affecter l'emphase qui est mise sur chaque rôle. Par exemple, la nature et la variété des rôles du dirigeant d'une PE n'appellerait d'un système formel qu'un accès facile à une information comptable simple et robuste (Dupuy, 1987). Le responsable de la fonction financière d'une ME dont l'une des tâches critiques est d'anticiper les problèmes de trésorerie (rôle «contrôleur») nécessiterait une capacité d'analyse d'informations plus complexes, incluant la simulation de flux de trésorerie à partir de prévisions de vente. Le responsable des opérations qui met l'emphase sur son rôle de «redresseur» aurait des besoins de repérage rapide et ponctuel d'informations d'exception sur la production, incluant les pénuries, les bris et les retards. Une telle approche pourrait s'opérationnaliser par une grille d'analyse des tâches critiques, spécifiant les caractéristiques de l'information et des capacités requises du SIO en fonction du rôle managérial associé à chaque tâches.

En fin de compte, le gestionnaire lui-même devrait être celui qui est le plus apte à concevoir un système d'information entrant en adéquation avec ses rôles décisionnels, et même interindividuels. Il s'agirait donc d'examiner les possibilités d'introduction de l'informatique de l'utilisateur final dans le contexte spécifique de la PME. Cela pourrait se faire par le biais «d'info-centres»(Brancheau, Vogel et Wetherbe, 1985), opérés par les organismes gouvernementaux, les universités ou les autres organismes qui offrent déjà des services de consultation aux PME. Les objectifs visés seraient de :

5 Dans une optique de développement «participatif», où les gestionnaires de PME s'impliquent activement dans l'analyse et la conception du SIO de leur entreprise (Raymond, 1987). 
- fournir des outils plutôt que des solutions, soit des logiciels de $4^{\text {me }}$ génération (tableurs, bases de données, logicielsintégrés, logicielsde bureautique)permettant au gestionnaire de concevoir sa propre solution à son problème de décision ou de communication;

- conseiller le gestionnaire sur l'utilisation des outils, en lui indiquant quel outil est approprié à quelle tâche (p.e. : tableur versus base de données);

- former le gestionnaire pour l'utilisation efficace d'un outil;

- offrir un service de consultation et d'assistance technique non seulement pour la conception, mais aussi pour la documentation, l'exploitation et la révision d'une application.

La promotion de ces services auprès des PME pourrait ainsi pallier aux lacunes actuelles des SIO en ce qui a trait aux rôles décisionnels et interindividuels des gestionnaires.

En terminant, nous devons mentionner certaines limites de la recherche. Compte tenu du caractère exploratoire de cette étude et de la nature de l'échantillon, les résultats ne peuvent pas nécessairement être généralisés à l'ensemble des gestionnaires de PME. De plus, le caractère ponctuel plutôt que longitudinal de l'étude ne nous permet pas de déterminer de véritables liens causals entre les variables de recherche. Nous espérons néanmoins que cette étude pourra servir de base inductive à des recherches futures.

\section{BIBLIOGRAPHIE}

ALEXANDER, L.D., The effect level in the hierarchy and functional area have on the extent Mintzberg's roles are required by managerial jobs, Academy of Management Proceedings, 1979, p. 186-189.

ALLOWAY, R.M. et QUILLARD, J.A., User managers' systems needs, Working Paper 86, Center for Information Systems Research, Massachusetts Institute of Technology, 1982.

BAILE, S., L'influence des facteurs de personnalité sur l'interaction homme-machine, Thèse d'Etat de Sciences de Gestion, Université de Montpellier I, 1985.

BARCET, A., BONAMY, J. et MAYERE, A., Informatique et PME : un dialogue différé, Economie et Humanisme, 1984, $\mathrm{n}^{\circ} 275$, pp. 44-56.

BRANCHEAU, J.C., VOGEL, D.R. et WETHERBE, J.C., An investigation of the information center from the user's perspective, Data Base, 1985, vol. 17, $\mathrm{n}^{\circ} 1$, p. 4-17.

CHICHA, J. et JULIEN, P.-A., Les stratégies des PME et leur adaptation au changement, Cahier de recherche 80-05, Groupe de recherche en économie et gestion des petites et moyennes organisations et de leur environnement, Université du Québec à Trois-Rivières, 1980. 
COOLEY, P.L., WALZ, D.T. et WALZ, D.B., A research agenda for computers and small business, American Journal of Small Business, 1987, vol. 11, n 3, p. 31-42.

CROZIER, M. et FRIEDBERG, E., L'acteur et le système, Paris, Seuil, 1977, p. 265-281.

CULNAN, M.J., Chauffered versus end user access to commercial databases : the effects of tasks and individual differences, MIS Quarterly, 1983, vol. 7, $\mathrm{n}^{\circ} 1, \mathrm{p} .55-67$.

DAVIS, G.D. et OLSON, M.H., Management Information Systems : Foundations, Structure and Development, New York, McGraw-Hill, 1985, p. 367-405.

DICKINSON, R.A.,FERGUSON, C.R. et SIRCAR, S., Critical success factors and small business, American Journal of Small Business, 1984, vol. 8, n 3, p. 49-57.

DICKSON, G.W., SENN, J.A. et CHERVANY, N.L., Research in management information systems : The Minnesota experiments, Management Science, 1977, vol. 23, no 9, p.913923.

DUPUY, Y., Vers de nouveaux systèmes d'information pour le chef de petite entreprise ?, Publication de recherche 87-2, Centre de recherche en gestion des organisations, I.A.E. de Montpellier, 1987.

FALLERY, B., Un système d'information pour les PME, Revue Française de Gestion, 1983, $n^{\circ} 43$, p. $70-76$.

GASSE, Y., Management techniques and practice in small manufacturing firms, Proceedings of the 24th Annual Conference of the International Council for Small Business, Québec, 1979, p. 16.1-16.15.

HARRELL, T.W., Differences between men in big and small business, Personnel Psychology, 1971, vol. 24, no 4, p. 649-652.

HENDERSON, J.C. et TREACY, M.E., Managing end-user computing for competitive advantage, Sloan Management Review, 1986, vol. 27, $\mathrm{n}^{\circ}$ 2, p. 3-14.

HOPPEN, N. et TRAHAND, J. , Les générateurs de systèmes d'aide à la décision pour la planification budgétaire et financière, Economies et Sociétés - Sciences de Gestion, 1985, $\mathrm{n}^{\circ}$ 6, p. 201-227.

KATZ, D. et KAHN, R.L., The Social Psychology of Organizations New York, John Wiley \& Sons, 1978, p. 188-189.

KERLINGER, F.N., Foundations of Behavioral Research, New York, Holt, Rinehart and Winston, 1973, p. 287.

KURKE, L.B. et ALDRICH, H.E. , Mintzberg was right : a replication and extension of the nature of managerial work, Management Science, 1983, vol. 29, $\mathrm{n}^{\circ} 8, \mathrm{p} .975-984$.

LEHMAN, J., Organizational size and information system sophistication, Working paper 85 18, MIS Research Center, University of Minnesota, 1985.

MASSEY, T.K. JR., Computers in small business : a case of under-utilization, American Journal of Small Business, 1986, vol. 11, $\mathrm{n}^{\circ} 2$, p. 51-60.

MINTZBERG, H., The Nature of Managerial Work, New York, Harper \& Row, 1973, p. 5499.

MINTZBERG, H., The myths of MIS, California Management Review, 1973, vol. $15, \mathrm{n}^{\circ} 1$, p. 92-97. 
MOORE, G.C., End user computing and office automation : a diffusion of innovations perspective, INFOR - Information Systems and Operational Research, 1987, vol. 25, $\mathrm{n}^{\circ} 3$, p. 214-235.

NICKELL, G.S. et P.C. SEADO, The impact of attitudes and experience on small business computer use, American Journal of Small Business, 1986, vol. 10, n 4, p. 37-48.

NIE, N.H. et Al., Statistical Package for the Social Sciences, New York, McGraw-Hill, 1975, p. 5,302 .

PAOLILLO, J.G.P.,The manager's self assessments of managerial roles : small vs large firms, American Journal of Small Business, 1984, vol. 8, $\mathrm{n}^{\circ} 3$, p. 58-64.

PAULSON, S.K. et STUMP, A.L., Small business and the theory of small bureaucracies, American Journal of Small Business, 1979, vol. 3, $\mathrm{n}^{\circ} 3$, p. 34-47.

POOLE, M.S., An information task approach to organizational communications, Academy of Management Review, 1978, vol. 3, n 3, p. 493-503.

RAYMOND, L., Organizational characteristics and MIS success in the context of small business, MIS Quarterly, 1985, vol. 9, n 1, p. 37-52.

RAYMOND, L., The impact of computer training on the attitudes and usage behavior of small business managers, Journal of Small Business Management, (à paraître), 1988.

RICE, A.W. et HAMILTON, R.E., Decision theory and the small businessman, American Journal of Small Business, 1979, vol. 4, $\mathrm{n}^{\circ}$ 1, p. 1-9.

SANDERS, G.L. et COURTNEY, J.F., A field study of organizational factors influencing DSS success, MIS Quarterly, 1985, vol. 9, n 1, p. 77-93.

SAUNDERS, C.S. et KELLER, R.T., A study of maturity of the information system function, task characteristics and interdepartmental communication : the importance of information system-organizational fit, Proceedings of the Fourth International Conference on Information Systems, Houston, 1983, p. 111-124.

SHAPIRA, Z. et DUNBAR, R.L.M., Testing Mintzberg's managerial roles classification using an in-basket simulation, Journal of Applied Psychology, 1980, vol. 65, $\mathrm{n}^{\circ} 1, \mathrm{p} .87$ 95.

SIMON, H.A., The New Science of Management Decision, New-York, Harper \& Row, 1960.

SNITKIN, S.R. et KING, W.R., Determinants of the effectiveness of personal decision support systems, Information \& Management, 1986, vol. 10, ${ }^{\circ} 2$, p. 83-89.

SRINIVASAN, A., Altemative measures of systems effectiveness : associations and implications, MIS Quarterly, 1985, vol. 9, n 3, p. 243-253.

TAYLOR, R.N. et DUNNETTE, M.D., Relative contribution of decision-maker attributes to decision processes, Organizational Behavior and Human Performance, 1974, vol. 12, $\mathrm{n}^{\circ} 2$, p. 286-298.

TIBBITS, G.E., Small business management : a normative approach, MSU Business Topics, 1979, vol. $27, \mathrm{n}^{\circ} 4$, p. 5-12.

TRAHAND, J., Informatique et fonction financière, Revue Française de Gestion, 1983, nº 43, p. 27-33. 
TRICE, A.W. et TREACY, M.E., Utilization as a dependent variable in MIS research, Proceedings of the Seventh International Conference on Information Systems, San Diego, 1986, p. 227-239.

YOUNG, E.C. et WELSCH, H.P., Information source selection patterns as determined by small business problems, American Journal of Small Business, 1983, vol. 7, $n^{\circ} 4$, p. 42 49.

ZINKHAN, G.M., JOACHIMSTHALER, E.A. et KINNEAR, T.C., Individual differences and marketing decision support system usage and satisfaction, Journal of Marketing Research, 1987, vol. 24, n 2, p. 208-214.

ZMUD, R.W., Individual differences and MIS success : a review of the empirical literature, Management Science, 1979, vol. 25, $\mathrm{n}^{\circ} 10$, p. 966-979. 